\title{
Secuencias de inserción IS6110 e IS1081 en cepas de Mycobacterium bovis provenientes de bovinos beneficiados en la Región Metropolitana
}

\author{
PATRICIO RETAMAL M. ${ }^{1}$, M. ANGÉLICA MARTÍNEZ T. ${ }^{2}$ y PEDRO ABALOS P. ${ }^{1}$
}

\section{Insertion sequences IS6110 and IS1081 present in strains of Mycobacterium bovis from cattle slaughtered at the Metropolitan Area, Chile}

Tuberculosis remains one of the major world health problems. Several studies have established the usefulness of insertion sequence (IS) IS6110 and IS1081 fingerprinting for the identification and typing of strains from the Mycobacterium tuberculosis complex. Both sequences are specific of bacteria belonging to this complex, but the number of copies can be variable among different $M$. tuberculosis and $M$. bovis strains. The aim of our study was to survey the presence of the insertion sequences IS6110 and IS1081 in nine strains of Mycobacterium bovis isolated from the mediastinal lymph nodes of cattle slaughtered in Santiago, Chile. All the strains contained IS1081, whereas only seven of them contained the IS6110 sequence. These results suggest the potential use of IS1081 for the differentiation by RFLP analysis of M. bovis strains. IS6110 may constitute an additional genetic marker for typing strains containing this element. It is recommended the characterization of this insertion sequences with genotyping assays, with higher sample sizes, more animal species and from different geographical areas to obtain useful epidemiological information in further control programs of the disease.

Key words: Mycobacterium bovis; Insertion sequence; Cattle.

\section{Introducción}

La tuberculosis fue declarada en 1993 como una emergencia global, estimándose en 1990 la ocurrencia de alrededor de 7,5 millones de enfermos y 2,5 millones de muertes en el mundo ${ }^{1,2}$. Aunque más de la mitad de los casos ocurre en África y en el sudeste asiático, en los países desarrollados y en aquellos en desarrollo se ha observado un cese en la declinación de la incidencia de la enfermedad ${ }^{1,2}$. La aparición y diseminación de cepas del complejo Mycobacterium tuberculosis multirresistentes a fármacos antituberculosos amenaza con dificultar su control ${ }^{1-3}$. Mycobacterium tuberculosis es el agente etiológico más frecuente de la tuberculosis humana. Mycobacterium bovis es el principal agente etiológico de tuberculosis bovina, pero tiene un amplio rango de hospederos incluido el hombre ${ }^{4}$. El conocimiento de la distribución de ambas especies es esencial para la identificación de reservorios animales potenciales que puedan constituir un riesgo para la salud pública, ya que se han descrito en el último tiempo brotes de tuberculosis humana causadas por cepas multirresistentes de $M$. bovis en pacientes infectados por $\mathrm{VIH}^{5-6}$. El desarrollo de técnicas moleculares basadas en la identificación y análisis de los ácidos nucleicos ha dado un gran impulso a la investigación epidemiológica de la tuberculosis humana y animal, al proporcionar herramientas que permiten identificar, comparar y trazar los patrones genómicos de cepas obtenidas en distintos escenarios epidemiológicos ${ }^{5-10}$. Entre los métodos de detección y genotipificación de estos microorganismos destaca por su utilidad epidemiológica el

\footnotetext{
Departamento de Medicina Preventiva Animal, Facultad de Ciencias Veterinarias y Pecuarias, Universidad de Chile.

Programa de Microbiología, Instituto de Ciencias Biomédicas, Facultad de Medicina, Universidad de Chile.
}

Recibido: 12 septiembre 2002

Aceptado: 7 julio 2003 
análisis del polimorfismo de la longitud de los fragmentos de restricción (PLFR, RFLP en sigla inglesa) con las secuencias de inserción IS6110 e IS10816-11. Ambas son específicas del complejo M. tuberculosis, pero su presencia y número de copias varía entre cepas de $M$. tuberculosis y $M$. bovis. Es así como IS6110 se encuentra en la mayoría de las cepas de $M$. tuberculosis y en mayor número de copias, mientras que IS 1081 se encuentra generalmente con mayor frecuencia y en mayor número en cepas de $M$. bovis ${ }^{6,7,9}$. En Chile, un estudio preliminar de genotipificación efectuado en 206 aislados de M. tuberculosis obtenidos de distintas regiones demostró la presencia de IS6110 en la totalidad de las cepas (Jorge Fernández, ISP, comunicación personal). No existen antecedentes nacionales de la presencia de estas secuencias de inserción en cepas de M. bovis.

El objetivo de nuestro estudio es comunicar los primeros hallazgos de la identificación de las secuencias de inserción IS6110 e IS1081 en cepas de $M$. bovis aisladas de bovinos beneficiados en la Región Metropolitana.

\section{Material y Método}

Cepas bacterianas. Se estudiaron 9 cepas de $M$. bovis aisladas de bovinos beneficiados en la Región Metropolitana entre julio y diciembre de 2001. Como controles se incluyeron las cepas de referencia $M$. tuberculosis $\mathrm{H} 37 \mathrm{Rv}$ y $M$. bovis BCG, aportadas gentilmente por el ISP de Chile. Para la obtención de estas 9 cepas se muestrearon 78 canales bovinas tipificadas en categorías $U$ y N. De cada una se extrajeron 20 a $40 \mathrm{~g}$ de un ganglio mediastinal durante su procesamiento en el matadero. El tratamiento de la muestra para el cultivo bacteriológico se realizó según el procedimiento descrito por Corner et $\mathrm{al}^{12}$. Para la descontaminación se utilizó hexadecilpiridina (HPC) al 0,25-0,5\%. Cada muestra se inoculó en duplicado en tubos de agar Middlebrook 7H10 (Becton Dickinson, EUA) sin glicerol, enriquecido con OADC (Becton Dickinson, EUA) y piruvato de sodio y en tubos de medio Stonebrink (ISPChile). Los cultivos fueron incubados a $25^{\circ} \mathrm{C}$ y a $37^{\circ} \mathrm{C}$, siendo observados semanalmente durante un período total de 12 semanas. El diagnóstico de M. bovis se efectuó por el aspecto morfológico, temperatura y velocidad de crecimiento de las colonias, y presencia de bacilos ácido alcohol resistente a la tinción de Ziehl-Neelsen ${ }^{12,13}$.

Reacción de polimerasa en cadena (RPC). Para la extracción del ADN, se preparó una suspensión de los cultivos en $200 \mathrm{ml}$ de PBS, la que fue inactivada a $80^{\circ} \mathrm{C}$ durante una hora. Las bacterias fueron posteriormente colectadas por centrifugación a $6.000 \mathrm{~g}$ durante $20 \mathrm{~min}$ y resuspendidas en $230 \mathrm{ml}$ de buffer TE $1 X$. Luego, las suspensiones bacterianas fueron tratadas con lisozima $(70 \mathrm{mg} / \mathrm{ml})$ a $37^{\circ} \mathrm{C}$ por $1 \mathrm{~h} \mathrm{y}$ posteriormente con SDS $(1 \%)$ y proteinasa $\mathrm{K}$ $(250 \mathrm{mg} / \mathrm{ml})$ a $65^{\circ} \mathrm{C}$ por $10 \mathrm{~min}$. El ADN fue finalmente purificado por el método de extracción con fenol cloroformo ${ }^{14}$.

Para la amplificación de IS1081 se utilizaron los partidores BW6 y BW7 descritos por Wards et $\mathrm{al}^{15}$, que amplifican un segmento de $306 \mathrm{pb}$ de la secuencia de ADN. Para la amplificación de IS6110 se utilizaron los partidores descritos por Eisenach et $\mathrm{al}^{16}$ que amplifican un segmento de $123 \mathrm{pb}$ de la secuencia. Ambas amplificaciones fueron efectuadas en volúmenes de $50 \mathrm{ml}$ conteniendo: buffer RPC $1 \mathrm{X}(50 \mathrm{mM} \mathrm{KCl}, 10 \mathrm{mM}$ Tris- $\mathrm{HCl}, \mathrm{pH} 8,3), 1,5 \mathrm{mM}$ de $\mathrm{MgCl}_{2}, 0,2 \mathrm{mM}$ de dNTPs, $1 \mathrm{mM}$ de los partidores y 2,5 unidades de Taq ADN polimerasa (Promega, EUA). El programa de amplificación consistió en una denaturación inicial a $95^{\circ} \mathrm{C}$ por $5 \mathrm{~min}$, seguido de 32 ciclos consistentes en denaturación a $95^{\circ} \mathrm{C}$ por $30 \mathrm{~s}$, anillaje a $68^{\circ} \mathrm{C}$ por $30 \mathrm{~s}$ y extensión a $72^{\circ} \mathrm{C}$ por $30 \mathrm{~s}$, para realizar una extensión final a $72^{\circ} \mathrm{C}$ por $5 \mathrm{~min}$. Los productos de la RPC fueron analizados por electroforesis en geles de agarosa al $1 \%$ teñidos con bromuro de etidio y visualización en transiluminador de luz ultravioleta, en comparación con marcadores de peso molecular.

\section{Resultados}

Se detectó la secuencia IS 1081 en la totalidad de las cepas de $M$. bovis y en las cepas controles M. bovis BCG y H37Rv. La secuencia IS6110 fue detectada en 7 de las cepas de $M$. bovis y en ambas cepas controles. En las Figuras 1 y 2 se observan los resultados de las amplificaciones de fragmentos de $306 \mathrm{pb}$ y 123 pb correspondientes a IS1081 e IS6110 respectivamente.

\section{Discusión}

El genoma de las especies del complejo $M$. tuberculosis se caracteriza por la presencia de numerosas secuencias de ADN repetidas, las que pueden ser empleadas para el análisis epidemiológico de los microorganismos ${ }^{8}$. La metodología de tipificación más utilizada, dada la gran homología del ADN existente entre las distintas 


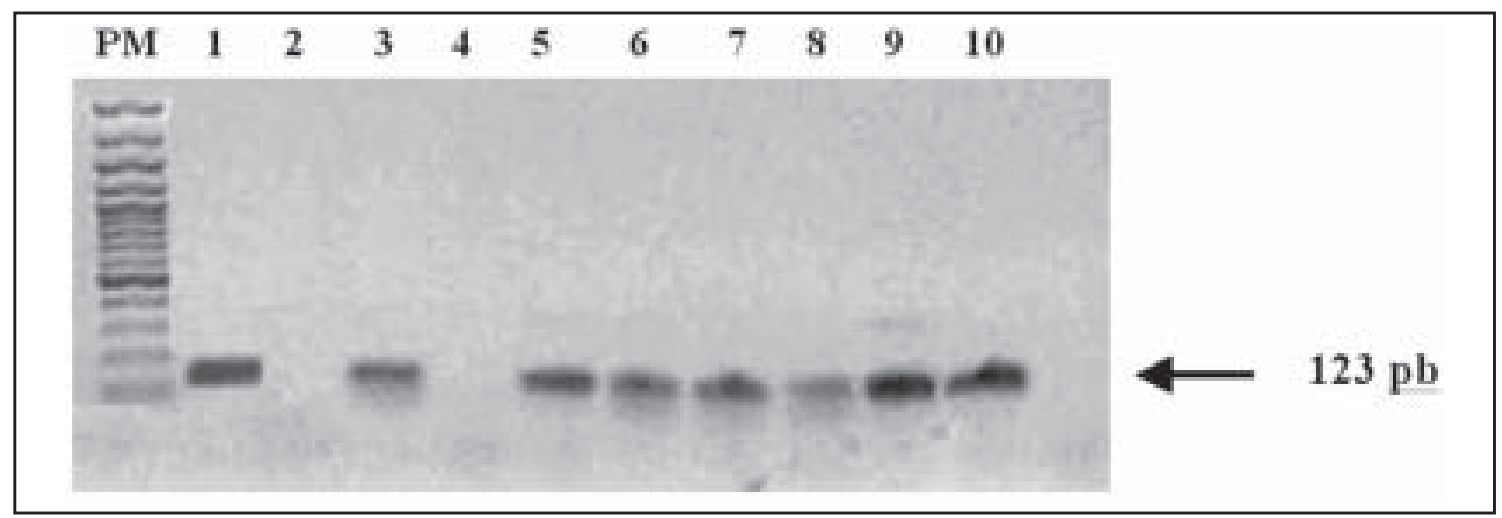

Figura 1. Electroforesis en gel de agarosa de la secuencia IS6110 amplificada por RPC desde cultivos de M. bovis. PM, marcador de peso molecular de 100 pb. Línea 1 representa a M. tuberculosis H37Rv. Líneas 2 a 10 representan a los aislados de M. bovis.

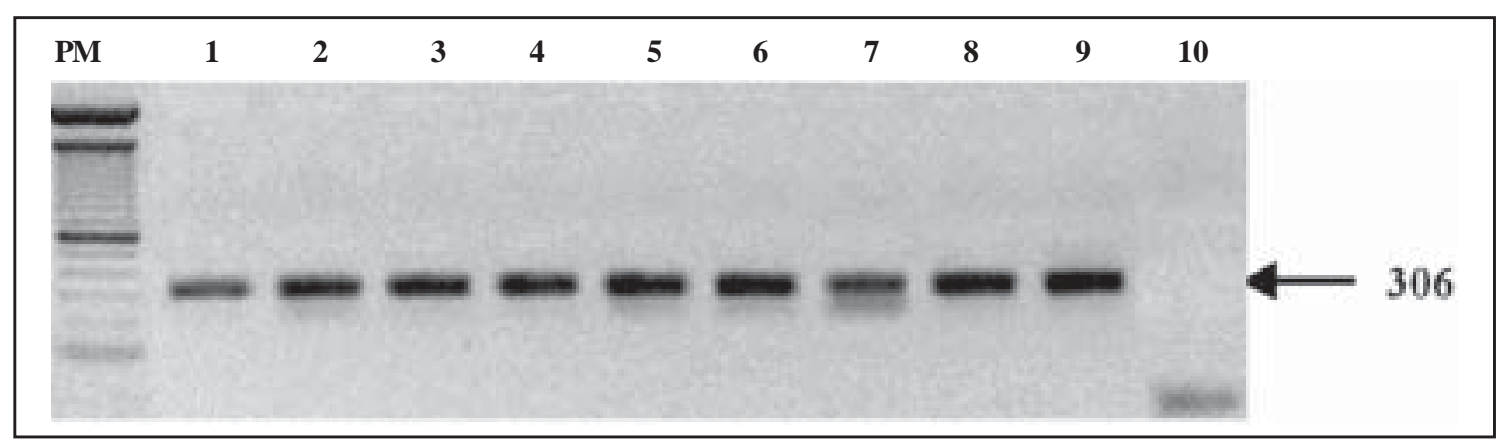

Figura 2. Electroforesis en gel de agarosa de la secuencia IS1081 amplificada por RPC desde cultivos de M. bovis. Líneas 1 a 9 representan a los aislados de $M$. bovis. Línea 10 corresponde a un control negativo (agua destilada).

especies del complejo $M$. tuberculosis, es el análisis del PLFR con estas secuencias repetidas. Existe un método estandarizado de PLFR con la secuencia de inserción IS6110 para la genotipificación de estos microorganismos ${ }^{15}$. Aunque existe una gran diversidad de patrones de PLFR-IS6110, estos tienden a parecerse entre cepas confinadas a una localidad geográfica, o aisladas desde brotes epidémicos con un origen común ${ }^{9}$. No obstante, se han descrito algunas cepas que no contienen esta secuencia de inserción o bien la presentan en un bajo número de copias, disminuyendo el poder de discriminación de PLFR-IS6110 y requiriendo la aplicación de técnicas adicionales para su adecuada genotipificación ${ }^{8-9}$. Por esta razón, en escenarios epidemiológicos como el nuestro, donde no se conocen las características genotípicas de las secuencias de inserción en la población de cepas circulantes, se necesitan estudios previos de estos agentes con el objeto de seleccionar las secuencias de ADN más apropiadas para la investigación epidemiológica molecular.
Nuestros resultados demuestran por primera vez en Chile, la existencia de cepas de $M$. bovis sin la secuencia IS6110 en bovinos beneficiados en un matadero de la Región Metropolitana. Estos hallazgos concuerdan con los resultados de estudios extranjeros que señalan que IS6110 está presente en un porcentaje variable de cepas de M. bovis ${ }^{16,17}$. IS1081 ha demostrado ser útil tanto para el diagnóstico de tuberculosis bovina como también en la tipificación de cepas de $M$. bovis ${ }^{11,17-20}$. Esta secuencia se ha utilizado en ensayos PLFR como complemento de otros procedimientos, permitiendo aumentar la discriminación de la genotipificación, y para identificar casos de la enfermedad donde $M$. bovis BCG ha sido la causa. IS1081 fue detectada en todas las cepas estudiadas, sugiriendo su potencial para ser usada como técnica de tipificación adicional de este microorganismo.

En los últimos años se ha observado un creciente aumento en la incidencia de tuberculosis humana causada por $M$. bovis en los países desarrollados. Se ha sugerido que el daño inmu- 
nológico ocasionado por la infección por el VIH favorecería la infección por este microorganismo, como asimismo la transmisión entre pacientes, dando origen a brotes institucionales ${ }^{5,6,21}$. Por este motivo es necesario investigar las posibles fuentes animales de infección humana. Por otra parte, la identificación de los genotipos comunes en una región estimularía el interés por conocer las características de las cepas con mayor potencial de diseminación o de mayor virulencia.

La tuberculosis bovina es una infección endémica en los ganados bovinos de América del Sur, especialmente en zonas donde la producción animal constituye una importante fuente de divisas, como Argentina y Brasil, ocasionando de esta forma importantes pérdidas económicas ${ }^{22}$. En Chile existe información epidemiológica limitada, donde el control de la enfermedad se basa en el diagnóstico de campo por la prueba de tuberculina $\mathrm{y}$ en los decomisos a nivel de mataderos por el examen post-mortem. La aplicación de técnicas moleculares para el diagnóstico y tipificación, más sensibles, específicas y rápidas que las pruebas tradicionales, permitirán mejorar el conocimiento de la epidemiología de la infección y facilitar las decisiones para su control.

En conclusión, los resultados de nuestro estudio sugieren la existencia de una proporción de cepas de $M$. bovis que carecen del elemento de inserción IS6110 y la utilidad potencial de la secuencia IS1081. El desafío actual es continuar la caracterización de estas secuencias de inserción con técnicas de genotipificación, estudiando un mayor número de cepas provenientes de distintas especies animales, incluyendo al ser humano, y de distintas zonas geográficas. La información epidemiológica generada de estos esfuerzos permitirán la planificación de mejores estrategias de control de la enfermedad, tanto en el ámbito de la salud humana como animal.

\section{Resumen}

La tuberculosis constituye uno de los principales problemas de salud pública en el mundo. Varios estudios han comunicado la utilidad de la técnica de fingerprint con las secuencias de inserción (IS) 6110 e IS1081 para la identificación y tipificación de cepas del complejo Mycobacterium tuberculosis. Ambas secuencias son específicas de las especies bacterianas que pertenecen a este complejo, pero su presencia y número de copias varía entre cepas de $M$. tuberculosis y M. bovis. El objetivo de nuestro estudio fue investigar la presencia de las secuencias
IS6110 e IS1081 en nueve cepas de Mycobacterium bovis aisladas de los ganglios mediastínicos de bovinos beneficiados en mataderos de Santiago de Chile. Se detectó la presencia de IS1081 en todas las cepas, mientras que IS6110 sólo fue detectada en 7 de ellas. Los resultados de este estudio sugieren la utilidad potencial de IS1081 para ser aplicada en la tipificación de cepas de $M$. bovis. La secuencia IS6110 podría constituir un marcador genético adicional en las cepas que contengan este elemento. Se requiere continuar la caracterización de estas secuencias de inserción con técnicas de genotipificación en que se incluya un mayor número de muestras, de distintas especies animales y provenientes de distintas zonas geográficas, a fin de generar información epidemiológica útil en futuros programas de control de la enfermedad.

\section{Bibliografía}

1.- Dolin P J, Raviglione M C, Kochi A. Global tuberculosis incidence and mortality during 1990-2000. Bull World Health Organ 1994; 72: 213-20.

2.- Raviglione M C, Snider D E, Kochi A. Global epidemiology of tuberculosis. Morbidity and mortality of a worldwide epidemic. JAMA 1995; 273: 220-6.

3.- Heymann S J, Brewer T F, Wilson M E, Fineberg H V. The need for global action against multidrug-resistant tuberculosis. JAMA 1999; 281: 2138-40.

4.- World Health Organization. Zoonotic tuberculosis (Mycobacterium bovis): memorandum from a WHO meeting. Bull World Health Organ 1994; 72: 851-7.

5.- Bouvet E, Casalino E, Mendoza-Sassi G et al. A nosocomial outbreak of multidrug-resistant Mycobacterium bovis among HIV-infected patients. A casecontrol study. AIDS 1993; 7: 1453-60.

6.- Blázquez J, Espinosa de Los Monteros L E, Samper S et al. Genetic characterization oí multidrug-resistant Mycobacterium bovis strains from a hospital outbreak involving human immunodeficiency virus-positive patients. J Clin Microbiol 1997; 35: 1390-3.

7.- Mazurek G H, Cave M D, Eisenach K D, Wallace R J, Bates J H, Crawford J T. Chromosomal DNA fingerprint patterns produced with IS6110 as strainspecific markers for epidemiologic study of tuberculosis. J Clin Microbiol 1991; 29: 2030-3.

8.- van Soolingen D, de Haas P E, Hermans P W, Groenen P M, van Embden J D. Comparison of various repetitive elements as genetic markers for strain differentiation and epidemiology of Mycobacterium tuberculosis. J Clin Microbiol 1993; 31: 1987-95.

9.- Yang Z, Barnes P F, Chaves F et al. Diversity of DNA fingerprinting of Mycobacterium tuberculosis isolates in the United States. J Clin Microbiol 1998; 36: 1003-7.

10.- Niemann S, Richter E, Rusch-Gerdes S. Stability of Mycobacterium tuberculosis IS6110 restriction fragment length polymorphism patterns and spoligotypes determined by analyzing serial isolates from patients with drug-resistant tuberculosis. J Clin Microbiol 1999; 37: 409-12. 
11.- Van Soolingen D, Hermans P W de Haas P E, van Embden J. Insertion element IS1081-associated restriction fragment length polymorphisms in Mycobacterium tuberculosis complex species: a reliable tool for recognizing Mycobacterium bovis BCG. J Clin Microbiol 1992; 30: 1772-7.

12.- Corner L A. Bovine tuberculosis: Pathology and Bacteriology. En Australian Standard Diagnostic Techniques for Animal Diseases. Corner LA and Bagust TJ (Eds). CSIRO for the Standing Committee on Agriculture and Resource Management: East Melbourne, 1993.

13.- Forbes B A, Sahm D F, Weissfeld A S Eds. Mycobacteria. En: Bailey \& Scott's Diagnostic Microbiology. Mosby, New York, 1998. 10 ${ }^{\text {th }}$ ed pp: 715-50.

14.- Graves L M, Swaminathan B. Universal bacterial DNA isolation procedure. En: Persing DH, Smith TF, Tenover FC, Wite TJ, Eds. Diagnostic Molecular Microbiology Principles and Applications. $1^{\text {st }}$ ed. Washington, DC: American Society for Microbiology, 1993.

15.- Wards B, Collins D, de Lisle G. Detection of Mycobacterium bovis in tissues by polymerase chain reaction. Vet Microbiol 1995; 43: 227-40.

16.- Eisenach K D, Sifford M D, Cave M D, Bates J H, Crawford J T. Detection of Mycobacterium tuberculosis in sputum samples using a polymerase chain reaction. Am J Respir Dis 1991; 144: 1160-3.

17.- Van Embden J D A, Cave M D, Crawford J T et al. Strain identification of Mycobacterium tuberculosis by DNA fingerprinting: recommendation for a standardized methodology. J Clin Microbiol 1993; 31: 406-9.

18.- Liébana E, Aranaz A, Domínguez L et al. The insertion element IS6110 is a powerful tool for DNA fingerprinting of Mycobacterium bovis isolates from cattle and goats in Spain. Vet Microbiol 1997; 54: 223-33.

19.- Cousins D, Skuce A, Razwala R, van Embden D. Towards a standardized approach to DNA fingerprinting of Mycobacterium bovis. Int J Tuberc Lung Dis 1998; 2: 471-8.

20.- Van Soolingen D, Hermans PWM, de Haas PEW, van Embden J D A. Insertion element IS1081-associated restriction fragment length polymorphism in $\mathrm{Myco-}$ bacterium tuberculosis complex species: a reliable tool for recognizing Mycobacterium bovis BCG. J Clin Microbiol 1992; 30: 1772-7.

21.- Grange J M, Daborn C, Cosivi O. HIV-related tuberculosis due to Mycobacterium bovis. Eur Respir J 1994; 7: $1564-6$

22.- Zumárraga M J, Martin C, Samper S et al. Usefulness of spoligotyping in molecular epidemiology of Mycobacterium bovis-related infections in South America J Clin Microbiol 1999; 37: 296-303.

Correspondencia a:

Patricio Retamal Merino

E-mail:pretamal@uchile.cl 